\title{
The paradoxical effect of aldosterone on cardiovascular outcome in maintenance hemodialysis patients
}

\author{
Sun Ryoung Choi ${ }^{1,2}$, Young-Ki Lee ${ }^{2,3}$, Hayne Cho Park ${ }^{2,3}$, Do Hyoung Kim ${ }^{2,3}$, AJin Cho ${ }^{2,3}$, Juhee Kim ${ }^{3}$, \\ Kyu Sang Yun ${ }^{2,3}$, Jung-Woo Noh ${ }^{2,3}$, Min-Kyung Kang ${ }^{4}$ \\ ${ }^{1}$ Department of Internal Medicine, Hallym University Dongtan Sacred Heart Hospital, Dongtan, Republic of Korea \\ ${ }^{2}$ Kidney Research Institute, Hallym University, Seoul, Republic of Korea \\ ${ }^{3}$ Department of Internal Medicine, Hallym University Kangnam Sacred Heart Hospital, Seoul, Republic of Korea \\ ${ }^{4}$ Department of Cardiology, Hallym University Kangnam Sacred Heart Hospital, Seoul, Republic of Korea
}

\begin{abstract}
Background: Patients with end-stage kidney disease face increased risk of cardiovascular events, and left ventricular diastolic dysfunction (LVDD) contributes to the high occurrence of cardiovascular mortality (CM). Although a high serum aldosterone (SALD) level is involved in the development of cardiovascular complications in the general population, this association is unclear in patients undergoing hemodialysis. We aimed to determine the impact of SALD on LVDD and CM among hemodialysis patients (HDPs).

Methods: We performed a prospective cohort study of maintenance HDPs without cardiovascular disease. The patients were divided into two groups according to the median level of SALD. All patients underwent baseline echocardiography to evaluate diastolic dysfunction (E/e' ratio > 15). The LVDD and CM rates were compared between the high and low aldosterone groups.

Results: We enrolled a total of 60 adult patients (mean age, $57.9 \pm 12.1$ years; males, 30.0\%). The low aldosterone group had an increased left ventricular diastolic dimension compared with the high aldosterone group (52.2 $\pm 8.4 \mathrm{~mm}$ vs. $50.3 \pm 5.2 \mathrm{~mm}$, respectively; $\mathrm{p}=0.03$ ). Low log-aldosterone (odds ratio [OR], 0.40; 95\% confidence interval [Cl], 0.19-0.86) and large left atrial dimension (OR, 1.31; 95\% Cl, 1.11-1.54) were independent risk factors for LVDD at baseline. In addition, Cox regression analysis demonstrated that low SALD was an independent predictor of CM in HDPs (hazard ratio, 0.46; 95\% Cl, 0.25-0.85; $p=0.01$ ) during follow-up.

Conclusion: Low SALD was not only associated with LVDD but was also an independent predictor of CM among HDPs regardless of their interdialytic weight gain.
\end{abstract}

Keywords: Aldosterone, Cardiovascular disease, Hemodialysis

\section{Introduction}

Among patients on hemodialysis (HD), cardiovascular (CV) mortality is the leading cause of death and accounts for almost $50 \%$ of deaths in these patients. The renin-angio- tensin-aldosterone system (RAAS) plays a significant role in the pathogenesis of vascular remodeling of the heart and systemic organs, in addition to its classical role in the maintenance of water-electrolyte balance, acid-base balance, and blood pressure regulation [1]. Aldosterone is the prima-

Received: April 25, 2021; Revised: July 17, 2021; Accepted: July 19, 2021

Correspondence: Young-Ki Lee

Department of Internal Medicine, Hallym University Kangnam Sacred Heart Hospital, 1 Singil-ro, Yeongdeungpo-gu, Seoul 07741, Korea.

E-mail:km2071@naver.com

ORCID: https://orcid.org/0000-0003-3464-6144

Copyright (C) 2022 by The Korean Society of Nephrology

(a) This is an Open Access article distributed under the terms of the Creative Commons Attribution Non-Commercial and No Derivatives License (http:// creativecommons.org/licenses/by-nc-nd/4.0/) which permits unrestricted non-commercial use, distribution of the material without any modifications, and reproduction in any medium, provided the original works properly cited. 
ry effector hormone of the RAAS and may have deleterious effects, including fibrosis, hypertrophy, and stimulation of smooth muscle cell proliferation and collagen production with a concurrent elevation in inflammatory and oxidative signaling [2]. Accordingly, aldosterone contributes to the development of myocardial hypertrophy and interstitial fibrosis, thereby promoting left ventricular diastolic dysfunction (LVDD) [3-5]. Likewise, it is actively involved in the development of proinflammatory and prothrombotic states, such as atherosclerotic CV disease [6].

Despite accumulating evidence supporting the positive correlation between aldosterone concentration and CV mortality in the general population as well as in patients with chronic kidney disease (CKD) [7-9], the results have been conflicting in patients undergoing HD. Interestingly, two previous studies reported the paradoxical causality between aldosterone and mortality among HD patients $[10,11]$. Left ventricular (LV) remodeling may play an important role in this inverse correlation. Therefore, we hypothesized that low serum aldosterone levels may be associated with LVDD and may increase the incidence of CV mortality in maintenance HD patients.

\section{Methods}

\section{Study population}

This study was conducted at Hallym University Kangnam Sacred Heart Hospital (Seoul, Korea). Ninety-five maintenance HD patients were eligible for the study and were followed up from March 2012 to February 2019. HD was performed three times weekly for four hours each session. Each patient underwent outpatient HD for at least three months. Patients with a history of CV disease and transplantation who had an active infection or a documented malignancy or those who failed to undergo an echocardiogram were excluded. Of the 95 enrolled patients, 33 had a previous history of CV disease, one had liver cirrhosis, and one was treated for hepatocellular carcinoma. The 60 remaining patients were included in this study. Patients were grouped based on their levels of serum aldosterone (Fig. 1). This prospective observational study was conducted in accordance with the principles contained in the Declaration of Helsinki and was approved by the Institutional Review Board of Hallym University Kangnam Sacred Hospital (No. 2010-05-33). Written informed consent was obtained from all participants.

\section{Clinical and biochemical parameters}

Baseline parameters including demographic, laboratory, and HD data were collected during the study enrollment process. CV disease included coronary artery, cerebrovascular, and peripheral vascular diseases, and CV mortality was defined as death attributable to CV disease. Blood samples to assess the serum aldosterone level were obtained before the midweek dialysis session to measure various markers using standard techniques after a 15-minute period of rest in the supine position [10]. The method for the quantitative determination of the LIAISON aldosterone assay is a competitive assay that uses sheep monoclonal antibodies to capture the aldosterone molecule (intraassay coefficient of variation, $2.1 \%-4.2 \%$; interassay coefficient of variation, 5.8\%-10.5\%) (DiaSorin Inc., Stillwater, MN, USA).

The body mass index (BMI) was calculated as the participants' dry weight in kilograms divided by their height in meters squared $\left(\mathrm{kg} / \mathrm{m}^{2}\right)$. The $\mathrm{Kt} / \mathrm{V}$ was determined using the logarithmic estimate of the Daugirdas method [12]. To estimate the volume status, the interdialytic weight gain (IDWG) was calculated as the mean value of weight gain after three consecutive HD sessions during the week of enrollment [13]. The dry weight was ascertained by clinical assessment at least monthly using chest X-rays. The ratio of absolute IDWG to the patient's dry weight was defined as the IDWG\%; an IDWG\% of $\geq 4.0$ was regarded as excessive [14]. Medical staff and dietitians advised the patients to limit their sodium intake to $<2.3 \mathrm{~g}$ per day to improve volume control [15]. Additionally, patients who were currently taking an angiotensin-converting enzyme inhibitor (ACEI) or an angiotensin receptor blocker (ARB) were divided into two groups according to the prescription drug dose. Those taking the maximum dose of ACEI or ARB were placed into the high-dose group, while those on lower doses made up the low group.

\section{Echocardiography}

Transthoracic echocardiograms were performed for baseline assessments on a nondialysis day within 1 month from the date of receipt of informed consent and were obtained by fundamental imaging (two-dimensional, M-mode, and 


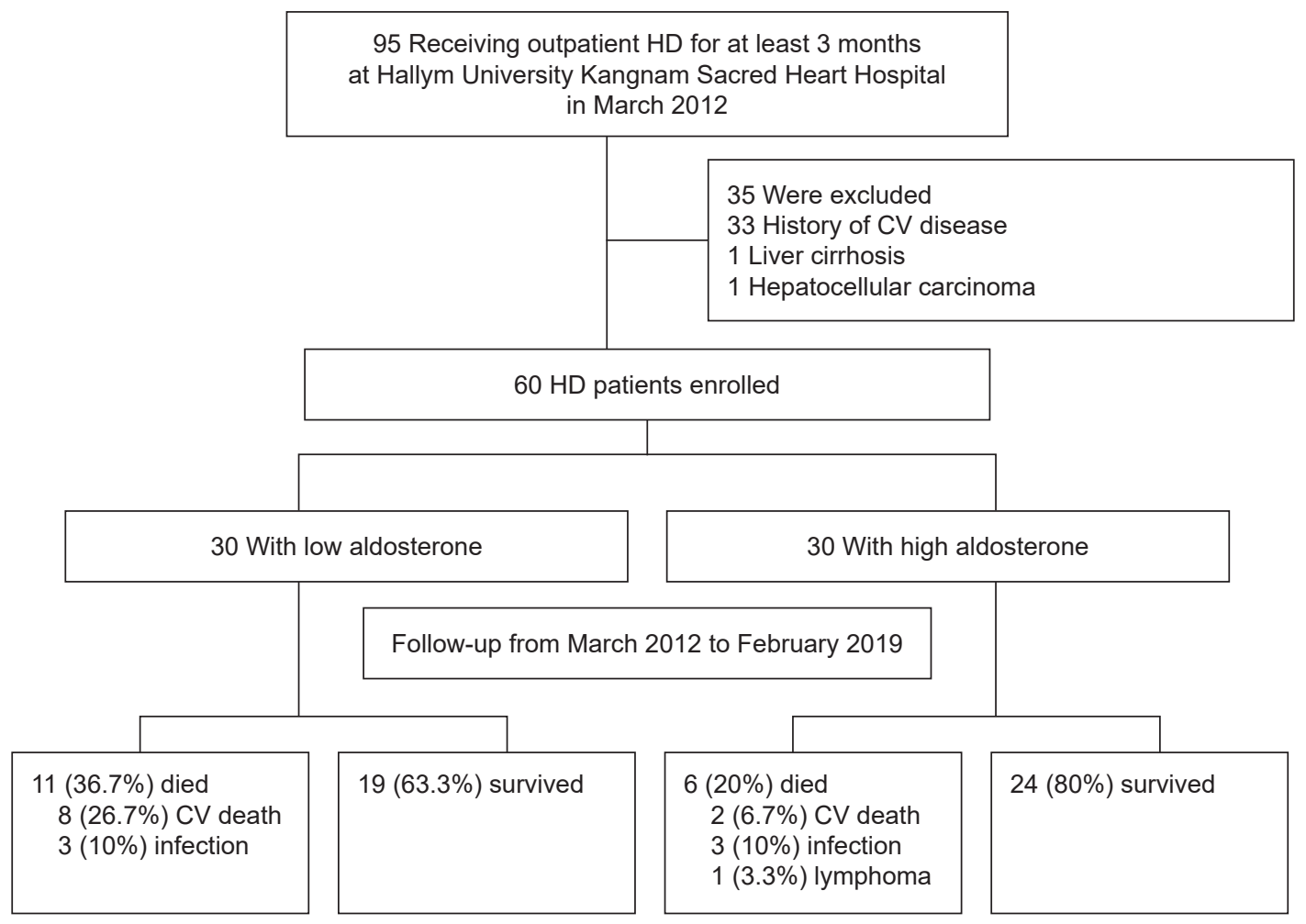

Figure 1. Flow diagram summarizing the process of patient enrollment and follow-up.

$\mathrm{HD}$, hemodialysis; CV, cardiovascular.

tissue Doppler imaging [TDI]) using a 2.5-MHz transducer and a commercial ultrasound system (Vivid 7; GE Vingmed Ultrasound AS, Horten, Norway); no further follow-up examinations were performed. The chamber dimensions, wall thickness, and LV ejection fraction (EF) were measured (M-mode), and the mitral annular velocities were obtained by TDI. The left atrial dimension (LAD) was determined from M-mode echocardiograms using a leading edge to leading edge technique by measuring the maximal distance between the posterior aortic root wall and the posterior LA wall at end-systole [16]. We measured the peak velocities of early diastole (e') and calculated the E/e' ratio, which reflects the mean LV diastolic pressure; an E/e' ratio of $>15$ indicates LVDD [17]. The LV mass was calculated using the Devereux formula $[\mathrm{LV}$ mass $(\mathrm{g})=1.04 \times$ (interventricular septal thickness + LV end-diastolic dimension + posterior wall thickness $)^{3}-(\text { LV end-diastolic dimension })^{3}-13.6$ [ [18], and the LV mass index (LVMI) was determined as the LV mass in grams divided by the body surface area. LV hypertrophy (LVH) was defined as an LVMI of at least $149 \mathrm{~g} / \mathrm{m}^{2}$ in males and $122 \mathrm{~g} / \mathrm{m}^{2}$ in females according to the current
American and European guidelines [19]. LV systolic function was assessed by calculating the EF using a modified Simpson's method, and LV systolic dysfunction (LVSD) was defined as an EF of $<50 \%$ [20]. Echocardiography was performed by two experienced specialists who were blinded to all patient information.

\section{Statistical analysis}

The patients were divided into two groups according to their median level of serum aldosterone. The associations between serum aldosterone, IDWG, and echocardiographic parameters were assessed at study enrollment. The participants were followed up to evaluate the effect of aldosterone on CV mortality until death or through March 2019. The researchers obtained the date and cause of death from a medical record review or a phone call at the end of the follow-up period. These summary statistics are expressed as the mean (standard deviation) or median (interquartile range) for continuous variables and as frequencies or percentages for categorical variables. Continuous variables were compared 
using the Student $t$ test for two groups or the Mann-Whitney $\mathrm{U}$ test for the nonparametric test. Categorical variables were compared using the chi-square test. A multivariate logistic regression analysis was performed to identify the independent risk factors for LVDD. A multivariate analysis that used the enter method was performed on variables that were shown to be meaningful upon univariate analysis. A Cox regression analysis was performed by the enter method to evaluate the influence of serum aldosterone on CV and allcause mortality. All tests were performed using the PASW Statistics version 18.0 (IBM Corp., Armonk, NY, USA). A p-value of $<0.05$ was considered statistically significant.

\section{Results}

Baseline characteristics according to serum aldosterone level

Among the 60 participants, the mean age was $57.9 \pm 12.1$ years (range, $34-82$ years), and 18 patients $(30.0 \%)$ were male. The median dialysis duration was 3.2 years (interquartile range, $0.4-22.0$ years). The underlying cause of end-stage renal disease (ESRD) was diabetes in 30 patients (50.0\%). Table 1 shows the baseline data of the low and high serum aldosterone groups. The high aldosterone group had higher levels of serum albumin $(4.1 \pm 0.3 \mathrm{~g} / \mathrm{dL}$ vs. $3.8 \pm 0.4 \mathrm{~g} / \mathrm{dL}$, respectively; $\mathrm{p}=0.008$ ) than the low aldosterone group. No significant differences were observed in the absolute IDWG ( 2.5 $\pm 1.3 \mathrm{~kg}$ vs. $2.4 \pm 1.1 \mathrm{~kg}$, respectively; $\mathrm{p}=0.88$ ), the proportion of excessive IDWG $(4.3 \% \pm 2.2 \%$ vs. $4.7 \% \pm 2.3 \%$, respectively; $\mathrm{p}=0.46)$, and the BMI $\left(23.2 \pm 3.4 \mathrm{~kg} / \mathrm{m}^{2}\right.$ vs. $21.5 \pm 3.1 \mathrm{~kg} / \mathrm{m}^{2}$, respectively; $\mathrm{p}=0.06$ ) between the high aldosterone group and the low aldosterone group (Table 1). There was no significant difference in the proportion of patients taking an ACEI or ARB between the two groups; likewise, there was no significant difference according to the dose.

\section{Baseline characteristics according to interdialytic weight gain}

Table 2 shows a comparative analysis of the baseline characteristics of patients with excessive IDWG and those without excessive IDWG. No significant difference was observed in the aldosterone levels between the group with an IDWG of $>4 \%$ and that without an IDWG of $>4 \%(\mathrm{p}=0.30)$ (Table 2$)$.
Patients with excessive IDWG had higher levels of serum potassium $(4.9 \pm 0.6 \mathrm{mEq} / \mathrm{L}$ vs. $4.3 \pm 0.7 \mathrm{mEq} / \mathrm{L}$, respectively; $\mathrm{p}=0.001)$, calcium $(8.5 \pm 1.0 \mathrm{mg} / \mathrm{dL}$ vs. $8.1 \pm 0.6 \mathrm{mg} / \mathrm{dL}$, respectively; $\mathrm{p}=0.03)$, phosphorus $(5.4 \pm 1.3 \mathrm{mg} / \mathrm{dL}$ vs. 4.1 $\pm 1.2 \mathrm{mg} / \mathrm{dL}$, respectively; $\mathrm{p}<0.001$ ), and high-density lipoprotein cholesterol $(46.9 \pm 15.1 \mathrm{mg} / \mathrm{dL}$ vs. $38.8 \pm 10.1 \mathrm{mg} /$ $\mathrm{dL}$, respectively; $\mathrm{p}=0.02)$ and lower levels of total $\mathrm{CO}_{2}(18.6$ $\pm 1.6 \mathrm{mmol} / \mathrm{L}$ vs. $20.7 \pm 2.3 \mathrm{mmol} / \mathrm{L}$, respectively; $\mathrm{p}<0.001$ ) than those without.

\section{Aldosterone as an independent risk factor for left ventric- ular diastolic dysfunction}

The log-aldosterone showed a negative correlation with the $\mathrm{E} / \mathrm{e}^{\prime}$ ratio $(\mathrm{r}=-0.435, \mathrm{p}=0.001)$ (Fig. 2$)$ and a significant positive relationship with the serum albumin $(\mathrm{r}=0.513, \mathrm{p}<$ 0.001 ). The low aldosterone group had a higher LV diastolic dimension than the high aldosterone group $(52.2 \pm 8.4 \mathrm{~mm}$ vs. $50.3 \pm 5.2 \mathrm{~mm}$, respectively; $\mathrm{p}=0.03$ ) (Table 3 ). The E/e' ratio $(21.0 \pm 11.9$ vs. $14.6 \pm 5.3, \mathrm{p}=0.01)$ and the proportion of LVDD ( $66.6 \%$ vs. $40.0 \%, \mathrm{p}=0.04$ ) were significantly higher in the low aldosterone group than in the high aldosterone group, respectively. There were no significant differences in LVMI and the proportion of LVH between the two groups. The high aldosterone group had higher EFs than the low aldosterone group. However, there was no significant difference in the proportion of LVSD between the two groups (Table 3). A multivariate logistic regression revealed that low log-aldosterone (odds ratio [OR], 0.40; 95\% confidence interval [CI], 0.19-0.86) and LAD (OR, 1.31; 95\% CI, 1.111.54) were independent risk factors for LVDD (Table 4).

\section{Aldosterone and predictors of cardiovascular mortality}

During the mean follow-up period of 5.2 years, 10 of 17 deaths were due to CV disease, which occurred in eight patients (26.7\%) in the low aldosterone vs. two patients (6.7\%) in the high aldosterone group (Fig. 1). A survival analysis showed that the cumulative incidence rates of CV mortality were significantly higher in the low aldosterone group (logrank test, $\mathrm{p}=0.03$ ) (Fig. 3). In a Cox multivariate analysis that had been adjusted for ACEI/ARB use, the albumin, high-sensitivity C-reactive protein (CRP), and Cox regression analysis showed that old age (OR, 1.17; 95\% CI, 1.061.30; $\mathrm{p}=0.003)$ and low aldosterone levels (OR, 0.46; 95\% 
Table 1. Baseline characteristics of patients according to serum aldosterone level

\begin{tabular}{|c|c|c|c|c|}
\hline Characteristic & All & Low aldosterone group & High aldosterone group & p-value \\
\hline \multicolumn{5}{|l|}{ Demographic data } \\
\hline No. of patients & 60 & 30 & 30 & \\
\hline Age (yr) & $57.9 \pm 12.1$ & $60.3 \pm 13.2$ & $55.5 \pm 10.6$ & 0.13 \\
\hline Male sex & $18(30.0)$ & $8(26.7)$ & $10(33.3)$ & 0.40 \\
\hline Diabetes mellitus & $30(50.0)$ & $15(50.0)$ & $15(50.0)$ & $>0.99$ \\
\hline Hypertension & $49(81.7)$ & $27(90.0)$ & $22(73.3)$ & 0.18 \\
\hline $\mathrm{SBP}(\mathrm{mmHg})$ & $143.4 \pm 11.4$ & $144.8 \pm 10.1$ & $141.9 \pm 12.6$ & 0.36 \\
\hline $\mathrm{DBP}(\mathrm{mmHg})$ & $82.1 \pm 7.2$ & $83.3 \pm 6.2$ & $80.7 \pm 7.9$ & 0.20 \\
\hline Dialysis vintage (yr) & $4.7 \pm 4.6$ & $4.0 \pm 3.8$ & $5.4 \pm 5.3$ & 0.25 \\
\hline Body mass index $\left(\mathrm{kg} / \mathrm{m}^{2}\right)$ & $22.3 \pm 3.4$ & $21.5 \pm 3.1$ & $23.2 \pm 3.4$ & 0.06 \\
\hline $\mathrm{Kt} / \mathrm{V}$ & $1.58 \pm 0.26$ & $1.58 \pm 0.21$ & $1.57 \pm 0.31$ & 0.85 \\
\hline \multicolumn{5}{|l|}{ Laboratory data } \\
\hline Log-aldosterone & $3.8 \pm 1.4$ & $2.8 \pm 1.2$ & $4.8 \pm 0.8$ & $<0.001$ \\
\hline Aldosterone $(\mathrm{ng} / \mathrm{dL})^{\mathrm{a}}$ & $44.0(0.1-1,210.4)$ & $23.0(0.1-44.0)$ & $96.8(47.5-1,210.4)$ & 0.001 \\
\hline Hemoglobin $(\mathrm{g} / \mathrm{dL})$ & $10.0 \pm 0.9$ & $9.8 \pm 0.8$ & $10.3 \pm 1.0$ & 0.07 \\
\hline hs-CRP (mg/L) & $2.1 \pm 1.9$ & $2.0 \pm 1.8$ & $2.2 \pm 2.1$ & 0.68 \\
\hline Albumin $(\mathrm{g} / \mathrm{dL})$ & $4.0 \pm 0.4$ & $3.8 \pm 0.4$ & $4.1 \pm 0.3$ & 0.008 \\
\hline iPTH (pg/mL) & $204.3 \pm 302.7$ & $197.9 \pm 384.9$ & $210.8 \pm 195.3$ & 0.87 \\
\hline Sodium (mEq/L) & $137.9 \pm 3.1$ & $137.9 \pm 3.2$ & $137.9 \pm 3.1$ & 0.94 \\
\hline Potassium (mEq/L) & $4.6 \pm 0.7$ & $4.5 \pm 0.7$ & $4.7 \pm 0.7$ & 0.13 \\
\hline Calcium (mg/dL) & $8.3 \pm 0.8$ & $8.2 \pm 0.7$ & $8.5 \pm 0.9$ & 0.28 \\
\hline Phosphorus (mg/dL) & $4.8 \pm 1.4$ & $4.6 \pm 1.3$ & $5.1 \pm 1.4$ & 0.18 \\
\hline Total $\mathrm{CO}_{2}(\mathrm{mmol} / \mathrm{L})$ & $19.5 \pm 2.1$ & $19.8 \pm 1.8$ & $19.1 \pm 2.4$ & 0.19 \\
\hline Total cholesterol (mg/dL) & $154.8 \pm 32.7$ & $150.1 \pm 26.9$ & $159.4 \pm 37.4$ & 0.28 \\
\hline Triglyceride (mg/dL) & $108.4 \pm 70.9$ & $91.5 \pm 51.8$ & $125.2 \pm 83.4$ & 0.07 \\
\hline HDL cholesterol (mg/dL) & $43.4 \pm 13.7$ & $43.1 \pm 12.5$ & $43.7 \pm 14.9$ & 0.86 \\
\hline LDL cholesterol (mg/dL) & $87.0 \pm 27.3$ & $85.5 \pm 20.9$ & $88.6 \pm 32.8$ & 0.66 \\
\hline \multicolumn{5}{|l|}{ Current medication $^{\mathrm{b}}$} \\
\hline Aspirin & $46(76.7)$ & $23(76.6)$ & $23(76.6)$ & $>0.99$ \\
\hline ACEI or ARB & $44(73.3)$ & $24(80.0)$ & $20(66.7)$ & 0.24 \\
\hline High dose & $28(63.7)$ & $17(70.8)$ & $11(55.0)$ & 0.35 \\
\hline Calcium channel blocker & $39(65.0)$ & $23(76.7)$ & $16(53.3)$ & 0.10 \\
\hline Beta-blocker & $33(55.0)$ & $20(66.7)$ & $13(43.3)$ & 0.12 \\
\hline Statin & $5(8.3)$ & $2(6.7)$ & $3(8.3)$ & $>0.99$ \\
\hline IDWG (kg) & $2.5 \pm 1.2$ & $2.4 \pm 1.1$ & $2.5 \pm 1.3$ & 0.88 \\
\hline IDWG\% & $4.5 \pm 2.2$ & $4.7 \pm 2.3$ & $4.3 \pm 2.2$ & 0.46 \\
\hline Excessive IDWG & $34(56.7)$ & $17(56.7)$ & $17(56.7)$ & $>0.99$ \\
\hline
\end{tabular}

Data are expressed as number only, mean \pm standard deviation, number (\%), or median (interquartile range).

ACEl, angiotensin-converting enzyme inhibitors; ARB, angiotensin receptor blocker; DBP, diastolic blood pressure; HDL, high-density lipoprotein; hs-CRP, high-sensitivity C-reactive protein; IDWG, interdialytic weight gain; iPTH, intact parathyroid hormone; LDL, low-density lipoprotein; SBP, systolic blood pressure.

${ }^{a}$ Median (interquartile range) values are presented because of a skewed distribution. ${ }^{b}$ None of the patients was treated with aldosterone antagonists.

CI, 0.25-0.85; $\mathrm{p}=0.01$ ) were significantly associated with $\mathrm{CV}$ mortality in maintenance HD patients (Table 5). However, the all-cause mortality $(\mathrm{p}=0.09)$ and non-CV mortality $(\mathrm{p}>$
0.99) rates were not significantly different between the two groups. 
Table 2. Comparative analysis of the baseline characteristics of patients according to IDWG

\begin{tabular}{|c|c|c|c|c|}
\hline Characteristic & All & IDWG/DW < 4\% & IDWG/DW $\geq 4 \%$ & p-value \\
\hline \multicolumn{5}{|l|}{ Demographic data } \\
\hline No. of patients & 60 & 26 & 34 & \\
\hline Age (yr) & $57.9 \pm 12.1$ & $60.5 \pm 13.1$ & $56.0 \pm 11.3$ & 0.16 \\
\hline Male sex & $18(30.0)$ & $10(38.5)$ & $8(23.5)$ & 0.17 \\
\hline Diabetes melliuts & $30(50.0)$ & $11(42.3)$ & 19 (55.9) & 0.22 \\
\hline Hypertension & $49(81.7)$ & $18(69.2)$ & $31(91.2)$ & 0.03 \\
\hline $\mathrm{SBP}(\mathrm{mmHg})$ & $143.4 \pm 11.4$ & $141.2 \pm 12.7$ & $145.4 \pm 10.0$ & 0.19 \\
\hline DBP (mmHg) & $82.1 \pm 7.2$ & $81.6 \pm 6.2$ & $82.5 \pm 8.0$ & 0.65 \\
\hline Dialysis vintage (yr) & $4.7 \pm 4.6$ & $3.5 \pm 4.4$ & $5.8 \pm 4.6$ & 0.06 \\
\hline Body mass index $\left(\mathrm{kg} / \mathrm{m}^{2}\right)$ & $22.3 \pm 3.4$ & $22.4 \pm 2.9$ & $22.4 \pm 3.8$ & 0.93 \\
\hline $\mathrm{Kt} / \mathrm{V}$ & $1.58 \pm 0.26$ & $1.52 \pm 0.28$ & $1.62 \pm 0.25$ & 0.15 \\
\hline \multicolumn{5}{|l|}{ Laboratory data } \\
\hline Log-aldosterone & $3.8 \pm 1.4$ & $3.7 \pm 1.5$ & $4.0 \pm 1.4$ & 0.30 \\
\hline Hemoglobin $(\mathrm{g} / \mathrm{dL})$ & $10.0 \pm 0.9$ & $10.3 \pm 1.1$ & $9.9 \pm 0.9$ & 0.13 \\
\hline hs-CRP (mg/L) & $2.1 \pm 1.9$ & $2.1 \pm 1.6$ & $2.2 \pm 2.2$ & 0.87 \\
\hline Albumin (g/dL) & $4.0 \pm 0.4$ & $3.9 \pm 0.5$ & $4.1 \pm 0.4$ & 0.23 \\
\hline iPTH (pg/mL) & $204.3 \pm 302.7$ & $153.4 \pm 120.3$ & $243.4 \pm 386.3$ & 0.26 \\
\hline Sodium (mEq/L) & $137.9 \pm 3.1$ & $138.4 \pm 3.1$ & $137.5 \pm 3.1$ & 0.30 \\
\hline Potassium (mEq/L) & $4.6 \pm 0.7$ & $4.3 \pm 0.7$ & $4.9 \pm 0.6$ & 0.001 \\
\hline Calcium (mg/dL) & $8.3 \pm 0.9$ & $8.1 \pm 0.6$ & $8.5 \pm 1.0$ & 0.03 \\
\hline Phosphorus (mg/dL) & $4.9 \pm 1.4$ & $4.1 \pm 1.2$ & $5.4 \pm 1.3$ & $<0.001$ \\
\hline Total $\mathrm{CO}_{2}(\mathrm{mmol} / \mathrm{L})$ & $19.5 \pm 2.1$ & $20.7 \pm 2.3$ & $18.6 \pm 1.6$ & $<0.001$ \\
\hline Total cholesterol (mg/dL) & $154.8 \pm 32.7$ & $160.6 \pm 37.0$ & $150.3 \pm 28.7$ & 0.23 \\
\hline Triglyceride (mg/dL) & $108.4 \pm 70.9$ & $140.4 \pm 76.9$ & $83.8 \pm 55.5$ & 0.002 \\
\hline HDL cholesterol (mg/dL) & $43.4 \pm 13.7$ & $38.8 \pm 10.1$ & $46.9 \pm 15.1$ & 0.02 \\
\hline LDL cholesterol (mg/dL) & $87.0 \pm 27.3$ & $93.6 \pm 29.8$ & $82.0 \pm 24.5$ & 0.10 \\
\hline \multicolumn{5}{|l|}{ Current medication } \\
\hline Aspirin & $46(76.7)$ & $18(69.2)$ & $28(82.4)$ & 0.23 \\
\hline ACEI or ARB & $44(73.3)$ & $15(57.6)$ & $29(85.3)$ & 0.02 \\
\hline Calcium channel blocker & $39(65.0)$ & $12(46.2)$ & $27(79.4)$ & 0.008 \\
\hline Beta-blocker & $33(55.0)$ & $6(23.1)$ & $27(79.4)$ & $<0.001$ \\
\hline Statin & $5(8.3)$ & $1(3.8)$ & $4(11.8)$ & 0.27 \\
\hline IDWG (kg) & $2.5 \pm 1.2$ & $1.4 \pm 0.8$ & $3.3 \pm 0.7$ & $<0.001$ \\
\hline IDWG\% & $4.5 \pm 2.2$ & $2.5 \pm 1.3$ & $6.1 \pm 1.5$ & $<0.001$ \\
\hline
\end{tabular}

Data are expressed as number only, mean \pm standard deviation, or number (\%).

ACEl, angiotensin-converting enzyme inhibitors; ARB, angiotensin receptor blocker; DBP, diastolic blood pressure; DW, dry weight; HDL, high-density lipoprotein; hs-CRP, high-sensitivity C-reactive protein; IDWG, interdialytic weight gain; iPTH, intact parathyroid hormone; LDL, low-density lipoprotein; SBP, systolic blood pressure.

\section{Discussion}

This study demonstrated the effect of low serum aldosterone on CV mortality irrespective of IDWG status. Low aldosterone level was significantly associated with a higher prevalence of LVDD. Moreover, low aldosterone was an independent predictor of CV mortality among patients on maintenance HD regardless of IDWG status. Our study results demonstrate the paradoxical effect of serum aldosterone on CV mortality in HD patients compared with the general population. An elevated serum aldosterone level is an established risk factor for $\mathrm{LVH}$ and myocardial fibrosis in hypertensive patients without ESRD [21]. However, the mechanisms behind this aldosterone paradox in HD patients are not well understood, and it has been argued that this association may be due to confounding variables. 

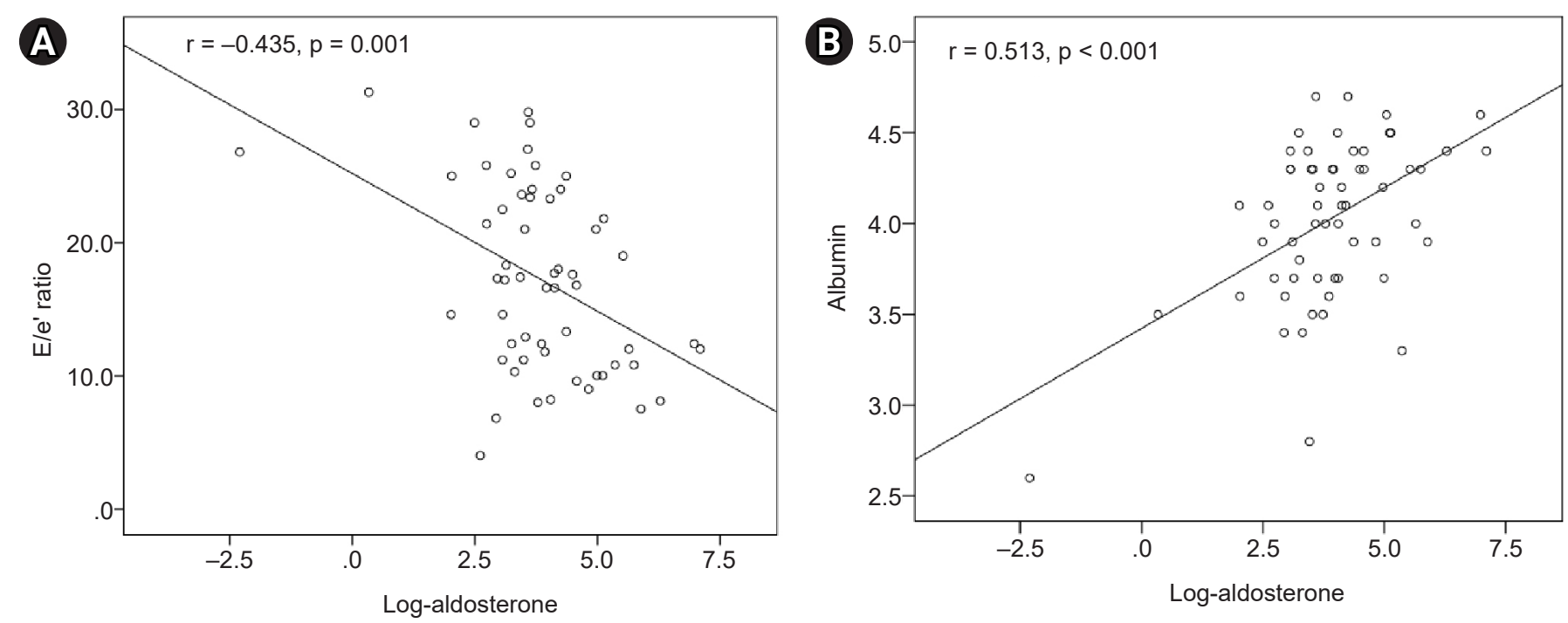

Figure 2. Correlations between log-aldosterone and $E / e^{\prime}$ ratio and log-aldosterone and serum albumin. $E / e^{\prime}$ presented a negative correlation $(r=-0.435, p=0.001)(A)$, while serum albumin demonstrated a positive correlation with log-aldosterone $(r=0.513$, $p<$ $0.001)(B)$.

Table 3. Baseline echocardiographic findings according to serum aldosterone level

\begin{tabular}{|c|c|c|c|c|}
\hline Variable & All $(n=60)$ & Low aldosterone group $(n=30)$ & High aldosterone group $(n=30)$ & p-value \\
\hline $\mathrm{LAD}(\mathrm{mm})$ & $41.6 \pm 7.3$ & $42.3 \pm 7.1$ & $41.2 \pm 6.7$ & 0.55 \\
\hline LAVI (mL) & $37.3 \pm 13.5$ & $40.8 \pm 15.1$ & $33.9 \pm 10.9$ & 0.05 \\
\hline $\operatorname{LVDd}(\mathrm{mm})$ & $51.2 \pm 7.0$ & $52.2 \pm 8.4$ & $50.3 \pm 5.2$ & 0.03 \\
\hline IVSd (mm) & $10.6 \pm 1.8$ & $10.6 \pm 1.6$ & $10.6 \pm 2.0$ & 0.89 \\
\hline $\mathrm{PWd}(\mathrm{mm})$ & $10.4 \pm 1.9$ & $10.6 \pm 1.6$ & $10.2 \pm 2.2$ & 0.43 \\
\hline $\mathrm{PAP}(\mathrm{mmHg})$ & $38.9 \pm 15.0$ & $41.2 \pm 15.3$ & $36.4 \pm 14.6$ & 0.23 \\
\hline LVMI $\left(g / \mathrm{m}^{2}\right)$ & $135.5 \pm 43.8$ & $141.6 \pm 44.7$ & $129.4 \pm 42.7$ & 0.29 \\
\hline $\mathrm{LVH}$ & $30(50.0)$ & $18(60.0)$ & $12(40.0)$ & 0.12 \\
\hline E/e' ratio & $17.2 \pm 6.9$ & $21.0 \pm 11.9$ & $14.6 \pm 5.3$ & 0.01 \\
\hline LVDD & $32(53.3)$ & $20(66.7)$ & $12(40.0)$ & 0.04 \\
\hline $\mathrm{EF}(\%)$ & $60.5 \pm 9.6$ & $57.2 \pm 10.9$ & $63.8 \pm 6.9$ & 0.007 \\
\hline LVSD & $5(8.3)$ & 4 (13.3) & $1(3.3)$ & 0.35 \\
\hline
\end{tabular}

Data are expressed as mean \pm standard deviation or number (\%).

LAD, left atrial dimension; LAVI, left atrial volume index; LVDd, end-diastolic left ventricular dimension; IVSd, interventricular septum thickness at end-diastole; PWd, posterior wall thickness at end-diastole; PAP, pulmonary artery pressure; LVMI, left ventricular mass index; LVH, left ventricular hypertrophy; E, early diastolic mitral inflow velocity; e', early diastolic mitral annular velocity; LVDD, left ventricular diastolic dysfunction; EF, ejection fraction; LVSD, left ventricular systolic dysfunction.

Aldosterone is metabolized predominantly in the liver and is excreted by the kidney. The serum concentration of aldosterone is substantially raised in HD patients. Cooke et al. [22] reported that no diurnal variation in the plasma aldosterone concentration could be demonstrated in HD patients. However, at least three factors play a role in the regulation of aldosterone secretion, including (1) sodium or volume-related stimuli mediated by the renin-angiotensin system, (2) potassium level, and (3) level of adrenocorticotropic hormone. Therefore, both the IDWG and sodium intake can be important triggers that lead to an increase in the plasma renin activity and aldosterone level. However, our study could not determine whether patients' pre-HD serum aldosterone levels can be a surrogate for long-term IDWG trends. As the glomerular filtration rate decreases, the plasma aldosterone level is inappropriately elevated relative to 
Table 4. Univariate and multivariate logistic regression analysis of LVDD

\begin{tabular}{|c|c|c|c|c|c|c|}
\hline & \multicolumn{3}{|c|}{ Univariate } & \multicolumn{3}{|c|}{ Multivariate } \\
\hline & Beta & OR $(95 \% \mathrm{Cl})$ & p-value & Beta & OR $(95 \% \mathrm{Cl})$ & p-value \\
\hline $\mathrm{LAD}(\mathrm{mm})$ & 0.251 & $1.29(1.14-1.46)$ & $<0.001$ & 0.268 & $1.31(1.11-1.54)$ & 0.001 \\
\hline Log-aldosterone & -0.648 & $0.52(0.31-0.88)$ & 0.02 & -0.910 & $0.40(0.19-0.86)$ & 0.02 \\
\hline Low aldosterone & 1.099 & $3.00(1.05-8.60)$ & 0.04 & & & \\
\hline LVMI $\left(\mathrm{g} / \mathrm{m}^{2}\right)$ & 0.023 & $1.02(1.01-1.04)$ & 0.005 & 0.004 & $1.00(0.98-1.03)$ & 0.74 \\
\hline Age (yr) & 0.029 & $1.03(0.99-1.08)$ & 0.20 & & & \\
\hline Hypertension & 0.847 & $2.33(0.60-9.02)$ & 0.22 & & & \\
\hline Diabetes mellitus & 0.815 & $2.26(0.80-6.36)$ & 0.12 & & & \\
\hline Dialysis vintage (yr) & 0.018 & $1.02(0.91-1.14)$ & 0.76 & & & \\
\hline Female sex & 0.838 & $2.31(0.75-7.16)$ & 0.15 & & & \\
\hline IDWG (kg) & 0.161 & $1.17(0.77-1.80)$ & 0.46 & & & \\
\hline Hemoglobin (g/dL) & -0.088 & $0.92(0.55-1.54)$ & 0.74 & & & \\
\hline Albumin (g/dL) & -0.198 & $0.82(0.25-2.68)$ & 0.74 & & & \\
\hline Phosphorus (mg/dL) & 0.193 & $1.21(0.83-1.77)$ & 0.31 & & & \\
\hline Use of ACEI/ARB & -0.526 & $0.59(0.19-1.87)$ & 0.37 & & & \\
\hline $\mathrm{SBP}(\mathrm{mmHg})$ & 0.038 & $1.04(0.99-1.09)$ & 0.14 & & & \\
\hline $\mathrm{DBP}(\mathrm{mmHg})$ & 0.024 & $1.03(0.95-1.11)$ & 0.54 & & & \\
\hline
\end{tabular}

ACEI, angiotensin-converting enzyme inhibitors; ARB, angiotensin receptor blocker; Cl, confidence interval; DBP, diastolic blood pressure; IDWG, intradialytic weight gain; LAD, left atrial dimension; LVDD, left ventricular diastolic dysfunction; LVMI, left ventricular mass index; OR, odds ratio; SBP, systolic blood pressure.

Covariates with p-values of $<0.05$ upon univariate analysis $(n=3)$ were included in the multivariate logistic analysis model, which used an enter method.

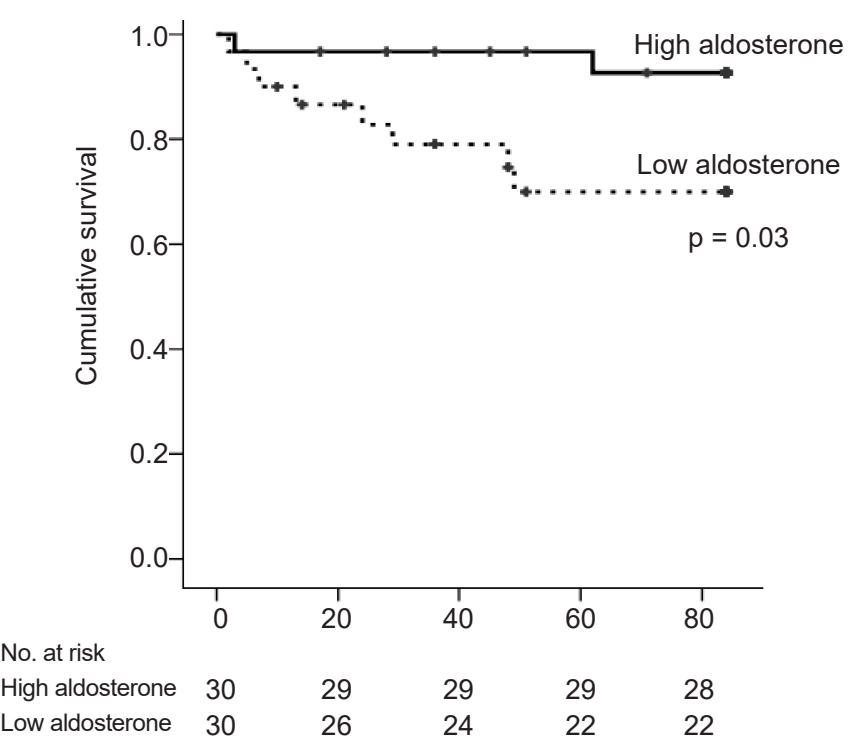

Figure 3. Kaplan-Meier survival curves for cardiovascular mortality according to the medians of log-aldosterone. Seven-year cardiovascular survival was significantly higher among patients in the upper median of log-aldosterone than those in the lower median of log-aldosterone $(93.3 \%$ vs. $73.3 \%$, log-rank test, $p=$ 0.027). the accompanying extracellular fluid expansion [23].

A growing body of evidence links aldosterone excess to an increased risk of CV mortality in the general population [7-9]. Patients with CKD also exhibit abnormally high aldosterone levels and a greater CV risk [24]. However, previous studies that have investigated the association between serum aldosterone and CV mortality in HD patients have yielded inconsistent results. Abd ElHafeez et al. [21] reported that the role of aldosterone as an inverse predictor of mortality was negligible after adjusting for potential confounders, such as malnutrition, inflammation, and volume biomarkers. In contrast, Drechsler et al. [25] found that elevated aldosterone levels were associated with strongly higher risks of sudden cardiac death in HD patients with diabetes. Our findings are consistent with those of Kohagura et al. [10], which showed that a lower aldosterone level was an independent predictor of mortality. They limited their study to hypertensive HD patients and excluded patients treated with RAAS inhibitors. In their study, the high aldosterone group had increased serum albumin and IDWG values and reduced CV and all-cause mortality rates. However, in our study, the high aldosterone group did not have either a higher IDWG or decreased non-CV mortality rates com- 
Table 5. Cox regression analysis of the predictors of cardiovascular mortality

\begin{tabular}{|c|c|c|c|c|}
\hline & \multicolumn{2}{|c|}{ Univariate } & \multicolumn{2}{|c|}{ Multivariate } \\
\hline & OR $(95 \% \mathrm{Cl})$ & p-value & OR $(95 \% \mathrm{Cl})$ & p-value \\
\hline Age (yr) & $1.14(1.06-1.22)$ & $<0.001$ & $1.17(1.06-1.30)$ & 0.003 \\
\hline Log-aldosterone & $0.53(0.37-0.76)$ & $<0.001$ & $0.46(0.25-0.85)$ & 0.01 \\
\hline Low aldosterone & $4.88(1.03-23.09)$ & 0.05 & & \\
\hline Albumin (g/dL) & $0.24(0.06-0.94)$ & 0.04 & $5.46(0.70-42.63)$ & 0.12 \\
\hline Phosphorus (mg/dL) & $0.62(0.39-0.99)$ & 0.05 & $1.10(0.59-2.08)$ & 0.76 \\
\hline LAD (mm) & $1.00(0.92-1.09)$ & 0.99 & & \\
\hline E/e' ratioe & $1.09(1.00-1.20)$ & 0.06 & & \\
\hline Hemoglobin $(\mathrm{g} / \mathrm{dL})$ & $0.90(0.48-1.68)$ & 0.73 & & \\
\hline hs-CRP (mg/L) & $1.19(0.93-1.54)$ & 0.20 & $1.24(0.91-1.69)$ & 0.17 \\
\hline Female sex & $1.90(0.40-8.96)$ & 0.42 & & \\
\hline Diabetes mellitus & $2.70(0.70-10.44)$ & 0.15 & & \\
\hline Hypertension & $1.01(0.21-4.75)$ & 0.99 & & \\
\hline $\mathrm{SBP}(\mathrm{mmHg})$ & $1.01(0.95-1.07)$ & 0.79 & & \\
\hline $\mathrm{DBP}(\mathrm{mmHg})$ & 0.99 (0.90-1.09) & 0.79 & & \\
\hline IDWG (kg) & $0.87(0.53-1.45)$ & 0.60 & & \\
\hline Dialysis vintage (yr) & 0.87 (0.69-1.09) & 0.22 & & \\
\hline Total cholesterol (mg/dL) & $0.97(0.98-1.02)$ & 0.97 & & \\
\hline Sodium (mEq/L) & $0.95(0.79-1.14)$ & 0.60 & & \\
\hline Potassium (mEq/L) & $0.54(0.23-1.25)$ & 0.15 & & \\
\hline Use of ACEI/ARB & $0.57(0.16-2.00)$ & 0.38 & $0.92(0.17-5.11)$ & 0.93 \\
\hline Use of CCB & $1.39(0.36-5.39)$ & 0.63 & & \\
\hline Use of a beta-blocker & $0.86(0.25-2.98)$ & 0.81 & & \\
\hline Use of a statin & $1.42(0.18-11.21)$ & 0.74 & & \\
\hline
\end{tabular}

ACEI, angiotensin-converting enzyme inhibitors; ARB, angiotensin receptor blocker; CCB, calcium channel blocker; Cl, confidence interval; DBP, diastolic blood pressure; E, early diastolic mitral inflow velocity; e`, early diastolic mitral annular velocity; hs-CRP, high-sensitivity C-reactive protein; IDWG, intradialytic weight gain; OR, odds ratio; SBP, systolic blood pressure.

Covariates with p-values $<0.05$ upon univariate analysis $(n=4)$, hs-CRP and use of ACEl/ARB were included in the multivariate logistic analysis model, method with enter.

pared with the low aldosterone group. Hung et al. [11] also demonstrated that a high aldosterone level was inversely associated with decreased all-cause mortality and CV event rates only during a state of volume overload. In contrast, our subgroup analysis of the presence of excessive IDWG did not show opposite survival curves ( $\mathrm{p}=0.79$, Supplementary Fig. 1A) and instead suggested that patients in the high aldosterone group without evidence of volume overload had better survival ( $100.0 \%$ vs. $69.2 \%, \mathrm{p}=0.03$ ) (Supplementary Fig. 1B). Contrary to our expectations, the inverse association of aldosterone levels became profound in patients without any volume overload. The difference between our findings and the results of the two studies $[10,11]$ mentioned above seemed to result from the differences in study population, the operational definition of volume overload, and the HD vintage. In our study, the group that did not have excessive IDWG had a tendency to have a shorter HD vintage, which may also have affected the results. Therefore, further investigation is needed to reveal the relationship among serum aldosterone, IDWG, and CV mortality.

LVDD develops early in most patients with cardiac diseases and leads to the elevation of LV filling pressure. Furthermore, it is an independent predictor of CV outcome. However, an inverse relationship between aldosterone and LVDD has not been established in HD patients. The prevalence and severity of LVDD gradually increase as renal function decreases, and it occurs in approximately $50 \%$ of HD patients, even in those without current symptoms of heart failure $[26,27]$. Similar to previous studies [28,29], our data showed that LVDD and LVSD were present in $53.3 \%$ and $8.3 \%$ of HD patients, respectively. Numerous studies have indicated that the $\mathrm{E} / \mathrm{e}^{\prime}$ ratio was the best noninvasive pre- 
dictor of an elevated LV filling pressure and LVDD [30,31]. Franczyk-Skóra et al. [26] showed that the E/e' ratio was two-fold higher in patients with stage 5 CKD than in those with stage $2 \mathrm{CKD}$, and it was also greater in patients on HD. In addition to the $\mathrm{E} / \mathrm{e}^{\prime}$ ratio, $\mathrm{LAD}$ is thought to reflect the $\mathrm{LV}$ filling pressure and has been considered an integrator of diastolic function over time. Therefore, this parameter provides diagnostic and prognostic information about LVDD and chronicity of disease [32]. Our data also support LAD as an independent risk factor for LVDD. By contrast, the main mechanism underlying LVDD is LVH with myocardial interstitial fibrosis, which induces myocardial stiffness and impairs heart function during diastole [33]. Among the complex pathophysiological factors, the RAAS plays a crucial role in cardiac hypertrophy, fibrosis, and inflammation [34]. However, our data demonstrated that low serum aldosterone level was associated with an increased risk of LVDD, which is not in accordance with the suggested mechanism.

The etiology of the reverse epidemiology between aldosterone and CV outcome in HD patients remains unclear, but several possible causes can be hypothesized. First, it may be related to a survival bias [35]. Only a small proportion of CKD patients undergo dialysis; therefore, it is likely that patients on HD have different CV risk factors. The factors that offer survival advantages to a small percentage of patients remain unknown. Another possible explanation is the malnutrition-inflammation complex. Serum albumin is recognized as a marker of malnutrition and inflammation and also is a strong predictor of CV outcome in HD [36]. A previous study [24] showed that aldosterone was inversely associated with inflammatory markers and directly associated with albumin, which suggested that the inflammation-protein wasting complex was the driving stimulus of aldosterone in HD patients. Similarly, the present study revealed a statistically significant positive relationship between serum aldosterone and albumin. Thus, aldosterone may be a nutritional and/or inflammatory marker, especially in chronic HD patients. Several studies have reported a possible association between inflammation and the pathophysiology of LVDD, although they did not include HD patients. Williams et al. [37] suggested that a higher CRP level was related to LVDD but not to systolic dysfunction. Matsubara et al. [38] also showed that inflammatory markers were elevated in patients with LVDD. More recently, Akin et al. [39] demonstrated a significant association between
LVDD and CRP elevation. The fact that the serum aldosterone level was higher in our study patients than in the general population [8] may imply that almost all HD patients are already exposed to CV risks. We do not presume that the serum aldosterone should be excluded from the CV risk factors within this population but instead propose that increased aldosterone may provide some survival advantages due to better nutrition and/or its postulated relationship with anti-inflammatory mediators. Third, IDWG may have been insufficient to adequately access patient volume status in this study. The clinical evaluation of volume status using IDWG has limitations since it does not necessarily correlate with extracellular fluid volume expansion, and chronic fluid overload could not be equivalent to IDWG [40].

This study had several limitations. First, this was a single-center study with small sample size. Second, an observational study may only provide an associative link but not a causative link; therefore, we cannot rule out the possibility of unmeasured confounding factors that influenced the implications between aldosterone and CV outcomes. Third, we did not perform bioimpedance spectroscopy to evaluate the patients' fluid status and did not evaluate the presence of residual renal function. In addition, adherence to a lowsalt diet was not assessed objectively. Lastly, we did not sequentially examine the echocardiographic parameters or the patients for the presence of CV events or risk factors (such as their nutritional, inflammatory, and volume status) during the follow-up period, which would have yielded more informative results.

In conclusion, this study demonstrated that low serum aldosterone level was not only inversely associated with LVDD but also was an independent predictor of CV mortality among maintenance HD patients without a previous history of CV disease regardless of IDWG. However, further research and clinical trials are needed to reveal the impact of aldosterone on cardiac function, malnutrition-inflammation complex, and CV outcomes in HD patients. Although aldosterone levels are influenced by several factors, elevated levels of serum aldosterone were not associated with an increase in $\mathrm{CV}$ mortality, at least in chronic $\mathrm{HD}$ patients.

\section{Conflicts of interest}

All authors have no conflicts of interest to declare. 


\section{Funding}

This study was supported by Baxter.

\section{Authors' contributions}

\section{Conceptualization: YKL, JWN \\ Data curation: JK, KSY \\ Formal analysis: SRC, HCP \\ Funding acquisition: YKL}

Investigation: SRC, HCP, YKL, MKK

Methodology: DHK, AC

Project administration: YKL

Visualization: SRC, HCP

Writing-Original Draft: SRC, HCP, YKL

Writing-Review \& Editing: All authors

All authors read and approved the final manuscript.

\section{ORCID}

Sun Ryoung Choi, https://orcid.org/0000-0002-9668-3349

Young-Ki Lee, https://orcid.org/0000-0003-3464-6144

Hayne Cho Park, https://orcid.org/0000-0002-1128-3750

Do Hyoung Kim, https://orcid.org/0000-0002-8664-8830

AJin Cho, https://orcid.org/0000-0001-7097-7026

Juhee Kim, https://orcid.org/0000-0002-2194-6327

Kyu Sang Yun, https://orcid.org/0000-0001-8019-3938

Jung-Woo Noh, https://orcid.org/0000-0002-1743-4695

Min-Kyung Kang, https://orcid.org/0000-0003-3838-951X

\section{References}

1. Waanders F, de Vries LV, van Goor H, et al. Aldosterone, from (patho)physiology to treatment in cardiovascular and renal damage. Curr Vasc Pharmacol 2011;9:594-605.

2. Gonçalves I, Edsfeldt A, Colhoun HM, et al. Association between renin and atherosclerotic burden in subjects with and without type 2 diabetes. BMC Cardiovasc Disord 2016;16:171.

3. Catena C, Colussi G, Brosolo G, Novello M, Sechi LA. Aldosterone and left ventricular remodeling. Horm Metab Res 2015;47: 981-986.

4. Schrier RW, Masoumi A, Elhassan E. Aldosterone: role in edematous disorders, hypertension, chronic renal failure, and metabolic syndrome. Clin J Am Soc Nephrol 2010;5:1132-1140.

5. Kritis AA, Gouta CP, Liaretidou EI, Kallaras KI. Latest aspects of aldosterone actions on the heart muscle. J Physiol Pharmacol 2016;67:21-30.

6. Grotevendt A, Wallaschofski H, Reincke M, et al. Associations of aldosterone and renin concentrations with inflammation-the Study of Health in Pomerania and the German Conn's Registry. Endocrine 2017;57:298-307.

7. Tomaschitz A, Pilz S, Ritz E, Meinitzer A, Boehm BO, März W. Plasma aldosterone levels are associated with increased cardiovascular mortality: the Ludwigshafen Risk and Cardiovascular Health (LURIC) study. Eur Heart J 2010;31:1237-1247.

8. Joseph JJ, Echouffo-Tcheugui JB, Kalyani RR, et al. Aldosterone, renin, cardiovascular events, and all-cause mortality among African Americans: The Jackson Heart Study. JACC Heart Fail 2017;5:642-651.

9. Vasan RS, Evans JC, Larson MG, et al. Serum aldosterone and the incidence of hypertension in nonhypertensive persons. $N$ Engl J Med 2004;351:33-41.

10. Kohagura K, Higashiuesato Y, Ishiki T, et al. Plasma aldosterone in hypertensive patients on chronic hemodialysis: distribution, determinants and impact on survival. Hypertens Res 2006;29:597-604.

11. Hung SC, Lin YP, Huang HL, Pu HF, Tarng DC. Aldosterone and mortality in hemodialysis patients: role of volume overload. PLoS One 2013;8:e57511.

12. Daugirdas JT, Depner TA, Greene T, Silisteanu P. Solute-solver: a web-based tool for modeling urea kinetics for a broad range of hemodialysis schedules in multiple patients. Am J Kidney Dis 2009;54:798-809.

13. Wong MM, McCullough KP, Bieber BA, et al. Interdialytic weight gain: trends, predictors, and associated outcomes in the International Dialysis Outcomes and Practice Patterns Study (DOPPS). Am J Kidney Dis 2017;69:367-379.

14. Lee MJ, Doh FM, Kim CH, et al. Interdialytic weight gain and cardiovascular outcome in incident hemodialysis patients. Am J Nephrol 2014;39:427-435.

15. Ikizler TA, Cano NJ, Franch H, et al. Prevention and treatment of protein energy wasting in chronic kidney disease patients: a consensus statement by the International Society of Renal Nutrition and Metabolism. Kidney Int 2013;84:1096-1107.

16. Sahn DJ, DeMaria A, Kisslo J, Weyman A. Recommendations regarding quantitation in M-mode echocardiography: results of a survey of echocardiographic measurements. Circulation 1978;58:1072-1083.

17. Kim JS, Yang JW, Yoo JS, Choi SO, Han BG. Association between E/e' ratio and fluid overload in patients with predialysis chronic 
kidney disease. PLoS One 2017;12:e0184764.

18. Hung CS, Chou CH, Wu XM, et al. Circulating tissue inhibitor of matrix metalloproteinase- 1 is associated with aldosterone-induced diastolic dysfunction. J Hypertens 2015;33:1922-1930.

19. Dietl A, Stark K, Zimmermann ME, et al. NT-proBNP predicts cardiovascular death in the general population independent of left ventricular mass and function: insights from a large population-based study with long-term follow-up. PLoS One 2016;11:e0164060.

20. Toida T, Toida R, Yamashita R, et al. Grading of left ventricular diastolic dysfunction with preserved systolic function by the 2016 American Society of Echocardiography/European Association of Cardiovascular Imaging Recommendations contributes to predicting cardiovascular events in hemodialysis patients. Cardiorenal Med 2019;9:190-200.

21. Abd ElHafeez S, Tripepi G, Mallamaci F, Zoccali C. Aldosterone, mortality, cardiovascular events and reverse epidemiology in end stage renal disease. Eur J Clin Invest 2015;45:1077-1086.

22. Cooke CR, Whelton PK, Moore MA, Caputo RA, Bledsoe T, Walker WG. Dissociation of the diurnal variation of aldosterone and cortisol in anephric subjects. Kidney Int 1979;15:669-675.

23. Schwenk MH, Hirsch JS, Bomback AS. Aldosterone blockade in CKD: emphasis on pharmacology. Adv Chronic Kidney Dis 2015;22:123-132.

24. Hené RJ, Boer P, Koomans HA, Mees EJ. Plasma aldosterone concentrations in chronic renal disease. Kidney Int 1982;21:98-101.

25. Drechsler C, Ritz E, Tomaschitz A, et al. Aldosterone and cortisol affect the risk of sudden cardiac death in haemodialysis patients. Eur Heart J 2013;34:578-587.

26. Franczyk-Skóra B, Gluba A, Olszewski R, Banach M, Rysz J. Heart function disturbances in chronic kidney disease: echocardiographic indices. Arch Med Sci 2014;10:1109-1116.

27. Kim JK, Kim SG, Kim MG, et al. Left ventricular diastolic dysfunction as a predictor of rapid decline of residual renal function in patients with peritoneal dialysis. J Am Soc Echocardiogr 2012;25:411-420.

28. Antlanger M, Aschauer S, Kopecky C, et al. Heart failure with preserved and reduced ejection fraction in hemodialysis patients: prevalence, disease prediction and prognosis. Kidney Blood Press Res 2017;42:165-176.

29. Jeong JH, Wu PT, Kistler BM, et al. The presence and impact of diastolic dysfunction on physical function and body composition in hemodialysis patients. J Nephrol 2015;28:739-747.

30. Nauta JF, Hummel YM, van der Meer P, Lam CS, Voors AA, van
Melle JP. Correlation with invasive left ventricular filling pressures and prognostic relevance of the echocardiographic diastolic parameters used in the 2016 ESC heart failure guidelines and in the 2016 ASE/EACVI recommendations: a systematic review in patients with heart failure with preserved ejection fraction. Eur J Heart Fail 2018;20:1303-1311.

31. Lassen MC, Biering-Sørensen SR, Olsen FJ, et al. Ratio of transmitral early filling velocity to early diastolic strain rate predicts long-term risk of cardiovascular morbidity and mortality in the general population. Eur Heart J 2019;40:518-525.

32. Mottram PM, Marwick TH. Assessment of diastolic function: what the general cardiologist needs to know. Heart 2005;91:681695.

33. Ogawa T, Nitta K. Clinical impact of left ventricular diastolic dysfunction in chronic kidney disease. Contrib Nephrol 2018;195:81-91.

34. Fukuta H, Goto T, Wakami K, Kamiya T, Ohte N. Effects of mineralocorticoid receptor antagonists on left ventricular diastolic function, exercise capacity, and quality of life in heart failure with preserved ejection fraction: a meta-analysis of randomized controlled trials. Heart Vessels 2019;34:597-606.

35. Kalantar-Zadeh K, Block G, Humphreys MH, Kopple JD. Reverse epidemiology of cardiovascular risk factors in maintenance dialysis patients. Kidney Int 2003;63:793-808.

36. Lacson E Jr, Wang W, Hakim RM, Teng M, Lazarus JM. Associates of mortality and hospitalization in hemodialysis: potentially actionable laboratory variables and vascular access. Am J Kidney Dis 2009;53:79-90.

37. Williams ES, Shah SJ, Ali S, Na BY, Schiller NB, Whooley MA. C-reactive protein, diastolic dysfunction, and risk of heart failure in patients with coronary disease: Heart and Soul Study. Eur $J$ Heart Fail 2008;10:63-69.

38. Matsubara J, Sugiyama S, Nozaki T, et al. Pentraxin 3 is a new inflammatory marker correlated with left ventricular diastolic dysfunction and heart failure with normal ejection fraction. $J$ Am Coll Cardiol 2011;57:861-869.

39. Akin F, Ayça B, Köse N, et al. Serum vitamin D and C-reactive protein levels are independently associated with diastolic dysfunction. J Investig Med 2014;62:43-48.

40. Hecking M, Moissl U, Genser B, et al. Greater fluid overload and lower interdialytic weight gain are independently associated with mortality in a large international hemodialysis population. Nephrol Dial Transplant 2018;33:1832-1842. 